\title{
Buckling Pneumatic Linear Actuators Inspired by Muscle
}

\section{Citation}

Yang, Dian, Mohit S. Verma, Ju-Hee So, Bobak Mosadegh, Christoph Keplinger, Benjamin Lee, Fatemeh Khashai, Elton Lossner, Zhigang Suo, and George M. Whitesides. 2016. Buckling Pneumatic Linear Actuators Inspired by Muscle. Advanced Materials Technologies 1, no. 3 : 1600055. Portico. doi:10.1002/admt.201600055.

\section{Published Version}

10.1002/admt.201600055

\section{Permanent link}

http://nrs.harvard.edu/urn-3:HUL.InstRepos:29951794

\section{Terms of Use}

This article was downloaded from Harvard University's DASH repository, and is made available under the terms and conditions applicable to Open Access Policy Articles, as set forth at http:// nrs.harvard.edu/urn-3:HUL.InstRepos:dash.current.terms-of-use\#OAP

\section{Share Your Story}

The Harvard community has made this article openly available.

Please share how this access benefits you. Submit a story.

\section{Accessibility}




\section{WILEY-VCH}

DOI: $10.1002 /(($ please add manuscript number $))$

Article type: Communication

\section{Buckling Pneumatic Linear Actuators Inspired by Muscle}

Dian Yang, Mohit S. Verma, Ju-Hee So, Bobak Mosadegh, Christoph Keplinger, Benjamin Lee, Fatemeh Khashai, Elton Lossner, Zhigang Suo, and George M. Whitesides*

D. Yang, Dr. M. S. Verma, Dr. J. So, Dr. B. Mosadegh, Dr. C. Keplinger, B. Lee, E. Khashai, E. Lossner, Prof. G. M. Whitesides

Department of Chemistry and Chemical Biology Harvard University, 12 Oxford Street, Cambridge, MA 02138, USA

E-mail: gwhitesides@gmwgroup.harvard.edu

Prof. Z. Suo, Prof. G. M. Whitesides

Kavli Institute for Bionano Science \& Technology Harvard University, 29 Oxford Street, Cambridge, MA 02138, USA

Dr. B. Mosadegh, Prof. G. M. Whitesides

Wyss Institute for Biologically Inspired Engineering Harvard University, 60 Oxford Street, Cambridge, MA 02138, USA

D. Yang, Prof. Z. Suo

School of Engineering and Applied Sciences Harvard University, 29 Oxford Street, Cambridge, MA 02138, USA

Keywords: buckling, soft actuators, pneumatic actuation, linear actuator, muscle-like actuator. 


\section{WILEY-VCH}

Soft actuators possess the ability to contact delicate, soft, and irregularly shaped objects (for example, fruit, animals, or clothing), because they distribute force across the surface of the objects, and because they are fabricated of compliant rather than unyielding materials. ${ }^{[1-8]}$ They also offer an attractive approach to simplifying control, ${ }^{[5-8]}$ since they make it possible —in some circumstances - to substitute the properties of soft materials and structures for some of the control loops, sensors, and actuators of hard machines (machines fabricated in metals, ceramics, and structural polymers). ${ }^{[9,10]}$ The simplification of control is important in the field of robotics as a potential strategy for designing robots that work in unstructured environments. ${ }^{[1]}$

Mechanical instabilities — here, the buckling of beams - are usually considered mechanisms of failure in hard structures. ${ }^{[12-14]}$ An emerging opportunity in the engineering of soft machines is to harness these instabilities to achieve new functionalities. ${ }^{[15-17]}$ For example, buckling has proven useful in the design of stretchable soft electronics, ${ }^{[18,19]}$ tunable materials, ${ }^{[20-}$ ${ }^{23]}$ and fluidic actuators. ${ }^{[24]}$ We have previously demonstrated that reversible buckling of elastomeric beams can be used to make a torsional soft actuator. ${ }^{[25]}$

This paper demonstrates that reversible buckling in assemblies of elastomeric beams can generate motions similar to those of skeletal muscles. Elastomeric pneumatic actuators based on buckling have, so far, demonstrated only rotation. ${ }^{[25]}$ The actuators we describe here- vacuumactuated muscle-inspired pneumatic structures (VAMPs) — differ from these previous elastomeric pneumatic actuators because they generates a linear motion, which resembles the motion of linear actuators in rigid robots (e.g., pistons and motor-actuated cables) and animals (e.g., muscle). VAMPs use buckling of elastomeric beams to generate muscle-like motions when negative pressure (vacuum) is applied to them; the external (ambient) pressure causes their cooperative, reversible collapse (that is, buckling). ${ }^{[26]}$ Like biological muscles, VAMPs are soft, 


\section{WILEY-VCH}

and thus able to absorb shock, and to interact non-destructively with their environment and collaboratively with humans. VAMPs also have a cellular structure, which has the potential to allow repair after damage. In contrast to previous soft actuators, which often move by expanding under pressure, VAMPs are similar to muscle in that they do not expand in cross-sectional area during actuation (although they contract in total volume); these geometrical features allow them to operate in space-constrained environments. The fact that VAMPs are made of elastomers allows them to store and release elastic energy. VAMPs can generate linear motions with strains up to $45 \%$, and can sustain loading stresses up to $65 \mathrm{kPa}$ (the theoretical maximum being 100 $\mathrm{kPa}$ under atmospheric pressure, with even higher values possible in hyperbaric environments); both strain and stress are similar to those of human muscle (typical strains of $\sim 20 \%$, with maximum values up to $\sim 40 \%$; maximum sustained stress of $\sim 100 \mathrm{kPa}$, and maximum impulsive stress of $\sim 350 \mathrm{kPa}){ }^{[27]}$ We also demonstrate that VAMPs made of Elastosil demonstrate a reasonable thermodynamic efficiency ( $27 \%$ efficiency at $20 \%$ strain without optimization); this value is within a factor of two of that of human muscle ( $\sim 40 \%$ efficiency). ${ }^{[27]}$

The history of muscle-like actuators is long. Among many such actuators, the McKibben actuator, developed in the 1950s, and its relatives (other braided muscle, knitted muscle, pleated muscle, etc. ${ }^{[28]}$ ) were the first truly successful examples. ${ }^{[29]}$ A typical McKibben actuator comprises a rubber balloon, constrained in volume by an inelastic mesh. On pressurization, the anisotropic inflation of this balloon results in useful motion. The properties of the mesh (i.e., the density of the weave, and the strength of the fibers) dictate the strain (typically $25 \%$ ) and stress (typically $800 \sim 1300 \mathrm{kPa}$ ) the actuator can produce for a given applied pneumatic pressure (typically $\sim 500 \mathrm{kPa}$ ) ${ }^{[30]}$ McKibben actuators have many practical applications, but have three characteristics that can limit their utility: ${ }^{[28]}$ i) Their inherent dry friction, and the non-elastic deformation of the mesh balloon, causes hysteresis; this hysteresis renders precise positional 


\section{WILEY-VCH}

control difficult. ii) They often cannot be actuated if the applied pressure is below a certain "threshold pressure"; this threshold may prevent the generation of low force. iii) The application of a pressure that is too high (typically $\sim 500 \mathrm{kPa}$ ) can make the balloon bulge through the mesh or at a point of damage, and perhaps burst. Other thoughtfully designed types of actuators (driven by electricity, temperature, and other means) have also been developed to mimic properties of the muscle. ${ }^{[27,31-33]}$

Actuators that operate based on negative pressure (vacuum) have been less explored than those using positive pressure, although they have two advantages: i) they cannot burst (nor can they "implode", as the actuation stops when the pneumatic chambers collapse to their minimum volume); ii) their volume decreases on application of vacuum (which allows them to function better in cluttered or space-constrained environments than actuators using positive pressure). Jaeger et al. described an example of this type of actuator, in which application of vacuum to an elastomeric bag filled with a granular solid caused jamming; this jamming increased the stiffness of the bag and its contents, and allowed gripping. ${ }^{[5]}$

Our research was stimulated by work of Bertoldi, Boyce, and colleagues, who demonstrated regular structural deformations in periodic elastomeric cellular solids—slabs of elastomer containing arrays of circular holes perpendicular to the slab-upon application of

mechanical force in the plane of the slab. ${ }^{[34,35]}$ Our structures are based on a different elastomeric structure that can be considered to comprise a number of interacting elastic beams and interconnected, deformable cavities sealed within a thin elastomeric membrane (Figure 1). Application of vacuum to the cavities causes the ambient atmospheric pressure to compress the device isotropically; this compression results in reversible, cooperative buckling of the beams, and in an anisotropic change in the shape of the structure. This design converts pressure-volume 


\section{WILEY-VCH}

work (done by applying negative pressure) into mechanical work (here, for example, lifting a weight).

The pattern of beams (or, equivalently, the pattern of voids in the elastomeric slab) we use is partially inspired by the auxetic structure discovered by Travesset, et al.- - a network of stiff levers that resembles the pattern of mortar in a brick wall. ${ }^{[36]}$ In one of our designs for VAMPs, the pattern consists of long, thin $(1.5 \mathrm{~mm})$ horizontal beams, bridged by thick $(4 \mathrm{~mm})$ vertical beams (Figure 1A; the SI and Figure S1 summarize details of fabrication; the SI also discusses how the behavior of the structure changes as the size of the features is increased and provides a modeling approach). When vacuum is applied to the structure, the horizontal beams buckle reversibly into serpentine shapes, and this buckling results in a large change in the vertical length of the structure $(\sim 40 \%)$, but a much smaller change $(\sim 5 \%)$ in its horizontal width (Figure 1B-D, Movies S1 and S2).

Static Mechanical Characteristics of VAMPs. We characterized two mechanical relationships for VAMPs (with dimension $34 \mathrm{~mm} \times 28 \mathrm{~mm} \times 46.5 \mathrm{~mm}$ ) under quasistatic conditions: those between i) applied differential pressure and change in length with no load; and ii) Young's modulus of the elastomer and the maximum load the structure can lift (that is, the force it can apply). We first define two variables: i) the difference in pressure, $\Delta P$, between the external ambient pressure Pext (typically $\sim 1 \mathrm{~atm} \approx 100 \mathrm{kPa}$ ) and the pressure inside the chambers Pint when under reduced pressure (Equation 1); and ii) the loading stress $\sigma$ (in $\mathrm{kPa}$ ) resulting from a weight applied to the VAMP (Equation 2). Here, $T$ is the force in the direction of actuation, and $A$ is the cross-sectional area of the undeformed actuator: 


\section{WILEY-VCH}

$$
\Delta P=P \text { ext }-P \text { int }
$$

$\sigma=T A$.

The actuation strain $s \Delta P, \sigma$ (i.e. the strain induced by actuation, rather than by external loading) is a function of these two variables (Equation 3), where $L(\Delta P, \sigma)$ is the length of the VAMP under loading stress $\sigma$ on application of a differential pressure $\Delta P$ :

$$
s \Delta P, \sigma=L \Delta P, \sigma-L 0, \sigma L 0,0 .
$$

Figure 2A, B shows the actuation strain of VAMPs under zero loading stress $s \Delta P, 0$. As the differential pressure $\Delta P$ increases, the VAMPs contract along their long axis, initially approximately linearly. Above a certain value ( $\Delta P$ crit) of $\Delta P$ (which depends on the properties of the elastomer), the VAMPs collapse almost completely. For Ecoflex 00-30 (Young's modulus $\mathrm{E}=43 \mathrm{kPa}), \Delta P$ crit is about $1 \mathrm{kPa}$; for Elastosil M4601 $(\mathrm{E}=520 \mathrm{kPa}), \Delta P$ crit is about $10 \mathrm{kPa}$. This critical differential pressure is proportional to the Young's modulus of the material (see the SI for a theoretical validation). Hence, VAMPs made of a material with critical Young's modulus $\mathrm{E} \approx 5 \mathrm{MPa}$ will have a $\Delta P$ crit of $\sim 100 \mathrm{kPa}(1 \mathrm{~atm})$, which is the largest pressure differential a vacuum system can provide at atmospheric pressure. This scaling rule describes the stiffness of the material of which VAMPs can be fabricated. In addition, the approximately linear relationship between the strain and applied pressure (for) shown in Figure 2A, B implies that VAMPs can stably ( $<1 \%$ change in height; details are in the SI) maintain any particular achievable strain given an applied pressure; this capability suggests that they should be easy to control. 


\section{WILEY-VCH}

Figure $2 \mathrm{C}, \mathrm{D}$ shows the actuation strain $s \Delta P, \sigma$ of VAMPs made of two different

elastomers as a function of loading stress $\sigma$, while applying a negative pressure greater than

$\Delta P$ crit. At a high differential pressure ( $\Delta P>\Delta P$ crit), increasing the loading stress $\sigma$ decreases the actuation strain $s \Delta P, \sigma$ of VAMPs, and the maximum loading stress is reached when the actuation strain is close to 0 . The maximum loading stress of VAMPs scales linearly with the Young's modulus of the actuator (see the SI for a theoretical validation; also see the SI for an explanation for the initial increase of actuation strain at low stress). A VAMP made of an elastomer with critical Young's Modulus $(\mathrm{E} \approx 5 \mathrm{MPa})$ has a projected loading stress of about 60 $\mathrm{kPa}$ at $30 \%$ actuation strain, and a loading stress of about $100 \mathrm{kPa}$ at $0 \%$ actuation strain. Figure S2 and Movie S3 show a VAMP that demonstrates $\sim 65 \mathrm{kPa}$ loading stress at $10 \%$ actuation strain when fabricated in a urethane elastomer with Young's modulus $\mathrm{E} \approx 2.5 \mathrm{MPa}$.

VAMPs made of materials with $\mathrm{E}>5 \mathrm{MPa}$ will not actuate fully under atmospheric pressure, but if Pext is greater (e.g. in hyperbaric environments such as undersea), much greater forces could be generated, using stiffer elastomers, and an actuation cycle that includes positive pressure to inflate the VAMP to $\Delta P=0$. The SI compares the force generated by VAMPs to biological muscle and other actuators.

We obtained the energy density of VAMPs fabricated in Elastosil (Figure S11) by using the data from the force vs. length plot of VAMPs (Figure 2D). For the VAMPs fabricated in Elastosil, the energy density peaks at $\sim 2 \mathrm{~J} / \mathrm{L}$ or $\sim 3.7 \mathrm{~J} / \mathrm{kg}$ (the density of VAMPs made of Elastosil is $\sim 0.54 \mathrm{~kg} / \mathrm{L}$, while the density of Elastosil itself is $\sim 1 \mathrm{~kg} / \mathrm{L}$ ). VAMPs made of 


\section{WILEY-VCH}

materials with critical Young's modulus are projected to demonstrate a peak work output per unit volume of $\sim 20 \mathrm{~J} / \mathrm{L}$.

Dynamic Mechanical Characteristics of VAMPs. We characterized the dynamic characteristics of VAMPs (fabricated in Elastosil, with dimension $34 \mathrm{~mm} \times 28 \mathrm{~mm} \times 46.5 \mathrm{~mm}$ ) by measuring their force-velocity relationships and power densities (the SI describes the method).

Figure 2E shows a force vs. velocity curve (derived from the raw displacement, velocity and acceleration data shown in Figure S10). The VAMPs demonstrate a faster actuation speed with a lighter load. We note that neither the force nor the velocity in this plot represent the theoretical maximum. Here, the force can be enhanced ten times by using an elastic material that is capable of large deformation and has ten times the Young's modulus of Elastosil, while the velocity can be enhanced by using pneumatic input with a larger radius.

Figure $2 \mathrm{~F}$ shows the power density of VAMPs as a function of velocity (derived from the force vs. velocity data in Figure 2E). For VAMPs fabricated in Elastosil, the power density peaks at $\sim 10 \mathrm{~W} / \mathrm{L}$ ( or $\sim 18.5 \mathrm{~W} / \mathrm{kg}$ ). VAMPs that are made of materials with critical Young's modulus, but maintain the same velocity, are projected to demonstrate a peak power output per unit volume of $\sim 100 \mathrm{~W} / \mathrm{L}$. The maximum power during isotonic contraction for natural skeletal muscles ranges from $7 \mathrm{~W} / \mathrm{kg}$ to $500 \mathrm{~W} / \mathrm{kg} .{ }^{[37]}$ As discussed previously, the velocity of these VAMPs is not at the physical limit. The SI compares the dynamic characteristic of VAMPs to that of biological muscle, and other various linear actuators. We also include in the SI a modeling approach to better analyze the dynamic characteristic of VAMPs (also see Figure S8). 


\section{WILEY-VCH}

Demonstrating Muscle-Like Motions. When vacuum is applied to their internal chambers, the principle contraction of VAMPs is uniaxial (with little change of cross-sectional area), in a direction determined by the pattern of their internal soft structures (Figure 1). These patterns can extend over substantial lengths and areas. Increasing the length increases the total displacement of the VAMPs on actuation, and increasing the cross-sectional area increases the force they generate (for example, in Figure 3 and Movies S4 and S5). In Figure 3A, the length of the VAMP decreased by $\sim 30 \%$ on actuation; the resulting displacement of the "hand" was about five times the change in length of the VAMP, because the lever arm ratio was 1:5 (a value similar to that of a human $\left.\operatorname{arm}^{[38]}\right)$. We also show that the speed of this "arm-like" structure driven by a VAMP is comparable to a human arm (see the SI, Figure S5, and Movies S6 and S7).

The relatively high speed of VAMPs allows them to perform tasks that are more dynamic than simple lifting (even without a complex control system). Figure S12 shows that the "armlike" structure can flip a cup in the palm of its "hand" into an upright position by using a jerking motion, which would not be possible with a slow muscle (see Movie S8). Figure S13 shows more demonstrations of the "arm-like" structure actuated by a VAMP in performing other muscle-like tasks (Movies S9 and S10). Humans and animals can perform more complex motions or sequences of motions by mobilizing a collection of many muscles and coordinating them with a neural network. By incorporating a collection of VAMPs of different sizes and coordinating them with an appropriate control system (not demonstrated in this work), biomimetic machines might imitate motions of more complex animal structures.

Demonstrating Features Unique to VAMPs. Being soft and actuated by vacuum, VAMPs possess many intrinsic functional traits that are not present in hard linear actuators, or in 


\section{WILEY-VCH}

pressure-actuated structures (e.g. McKibben actuators). Examples include functional traits such as compliance, shock absorbance, non-damaging interaction with the environment, and elastic energy storage (see the SI and Figure S7). Vacuum actuation allows VAMPs to be installed in space-limited positions (such as inside a rigid pipe). VAMPs also preserve their function and performance even after punctures. For example, the actuation was unchanged after a VAMP was punctured with a $2 \mathrm{~mm}$-wide metal cannula (Figure S6 and Movie S11). We attribute this selfsealing ability of VAMPs to compression around the puncture by atmospheric pressure and elastomeric retraction.

Thermodynamic Efficiency of VAMPS. The thermodynamic efficiency of transduction of the pressure-volume work required to actuate VAMPs into mechanical (force $\times$ distance) work (e.g., lifting a weight) is limited by the work required to compress the elastomer. By comparison, the loss of energy due to hysteresis is small (details are in the SI, Figure S3). Our experimental data gave a thermodynamic efficiency of $27 \%$ for an actuation strain of $20 \%$ at 500 -g loading for the VAMP shown in Figure 1D (details of the method are in the SI). For comparison, the corresponding value of a human skeletal muscle is $\sim 40 \% .{ }^{[27]}$ The energy stored in the deformed, elastomeric components (which is not converted into useful mechanical work and thus reduces the thermodynamic efficiency for a single, unidirectional motion) can, in principle, be at least partially recovered during unloading. A system containing VAMPs can, therefore, have a greater efficiency over multiple actuation cycles than that measured in a single cycle. (This method of storing and recovering energy of soft actuators has been reported in a number of designs ${ }^{[27]}$ ). 


\section{WILEY-VCH}

Conclusions. The mechanism by which VAMPs achieved their mechanical performance demonstrates a new approach for biomimetic actuation: nonlinear motion reflecting the cooperative, reversible, buckling and collapse of elastomeric beams. VAMPs have eight characteristics that will be useful in soft machines: i) no expansion in volume on contraction; ii) many aspects of mechanical performance on actuation similar to human muscle; iii) they are "collaborative" (to use the term common in robotics) — that is, safe in use around humans; iv) thermodynamic efficiency comparable to (although currently less than) muscle; v) a cost of fabrication sufficiently low that they can be considered for single-use applications; vi) performance that is reliable even after a high number of actuation cycles (VAMPs fabricated in Elastosil demonstrated no significant change in performance after a million cycles of actuation; SI, Figure S4); vii) light weight and low density (relative to metals); viii) properties characteristic of organic polymers (transparency, electrical properties that extend from insulating to conducting, biocompatibility, controllable rates of environmental degradation, ease of fabrication in complex shapes) that will be useful in specific applications.

VAMPs are not (and not intended to be) competing with hard actuators. They are complimentary, and intended largely for non-competitive and hybrid uses: examples would include assistive devices for the elderly or disabled, systems for non-damaging manipulation of irregularly shaped and delicate objects (fruit, tissues, small animals), devices intended to be inexpensive enough for single use (e.g., in search and rescue), or where resistance to damage by impact are important, and others where soft systems offer capabilities not easily or inexpensively embedded in hard systems with typical designs.

The designs of the actuators used in Figures 1 and 3 are a small subset of those that can be created through appropriate design and segmentation of the patterns of pillars making up the VAMPs, and through extension of these quasi-two-dimensional structures into three dimensions. 


\section{WILEY-VCH}

The key feature of all of these designs is, however, the use of cooperative, reversible buckling in an elastomeric material to achieve actuation. This work thus exploits buckling to achieve useful and simple (quasi-linear) motion through a mechanism that involves complex, nonlinear, cooperative motions of the internal structure of the VAMPs.

\section{Supporting Information}

Supporting Information is available from the Wiley Online Library or from the author.

\section{Acknowledgements}

Work on biomimetic design was funded by a subcontract from Northwestern University under DOE award number DE-SC0000989. Work on thermodynamics and mechanics was funded by the DOE, Division of Materials Sciences and Engineering, grant number ER45852. MSV is funded by the Banting Postdoctoral Fellowship from the Government of Canada. BM and CK acknowledge the Wyss Institute for Biologically Inspired Engineering for partial salary support. BL was supported by a Harvard University work-study program for undergraduates. FK was supported by the NSF-funded REU program under award number DMR-0820484.

Received: ((will be filled in by the editorial staff))

Revised: ((will be filled in by the editorial staff))

Published online: ((will be filled in by the editorial staff))

[1] D. Rus, M. T. Tolley, Nature 2015, 521, 467. 
[2] B. Trimmer, Curr. Biol. 2013, 23, R639.

[3] S. Kim, C. Laschi, B. Trimmer, Trends Biotechnol. 2013, 31, 287.

[4] K. Suzumori, S. Iikura, H. Tanaka, "Flexible Microactuator for Miniature Robots", presented at Proc. IEEE Int. Conf. Rob. Autom., 1991.

[5] E. Brown, N. Rodenberg, J. Amend, A. Mozeika, E. Steltz, M. R. Zakin, H. Lipson, H.

M. Jaeger, Proc. Natl. Acad. Sci. U.S.A. 2010, 107, 18809.

[6] F. Ilievski, A. D. Mazzeo, R. F. Shepherd, X. Chen, G. M. Whitesides, Angew. Chem. Int. Ed. 2011, 123, 1930.

[7] R. V. Martinez, J. L. Branch, C. R. Fish, L. Jin, R. F. Shepherd, R. Nunes, Z. Suo, G. M. Whitesides, Adv. Mater. 2013, 25, 205.

[8] B. Mosadegh, P. Polygerinos, C. Keplinger, S. Wennstedt, R. F. Shepherd, U. Gupta, J. Shim, K. Bertoldi, C. J. Walsh, G. M. Whitesides, Adv. Funct. Mater. 2014, 24, 2163.

[9] C. C. De Wit, G. Bastin, B. Siciliano, Theory of Robot Control, Springer-Verlag: New York, 1996.

[10] M. W. Spong, S. Hutchinson, M. Vidyasagar, Robot Modeling and Control, Vol. 3, Wiley: New York, 2006.

[11] C. C. Kemp, A. Edsinger, E. Torres-Jara, IEEE Robotics and Automation Magazine 2007, $14,20$.

[12] R. Bruzek, L. Biess, L. Al-Nazer, "Development of Rail Temperature Predictions to Minimize Risk of Track Buckle Derailments", presented at Proc. ASME Jt. Rail Conf., 2013.

[13] J. Gordo, C. Guedes Soares, D. Faulkner, J. Ship Res. 1996, 40, 60.

[14] J. Miller, T. Su, J. Pabon, N. Wicks, K. Bertoldi, P. Reis, Int. J. Solids. Struct. 2015, 72, 153.

[15] S. Singamaneni, V. V. Tsukruk, Soft Matter 2010, 6, 5681. 


\section{WILEY-VCH}

[16] D. Chen, J. Yoon, D. Chandra, A. J. Crosby, R. C. Hayward, J. Polym. Sci., Part B:

Polym. Phys. 2014, 52, 1441.

[17] N. Hu, R. Burgueño, Smart Mater. Struct. 2015, 24, 063001.

[18] J. A. Rogers, T. Someya, Y. Huang, Science 2010, 327, 1603.

[19] Y. Wang, R. Yang, Z. Shi, L. Zhang, D. Shi, E. Wang, G. Zhang, ACS Nano 2011, 5, 3645.

[20] J. Shim, C. Perdigou, E. R. Chen, K. Bertoldi, P. M. Reis, Proc. Natl. Acad. Sci. U.S.A. 2012, 109, 5978.

[21] B. Florijn, C. Coulais, M. van Hecke, Phys. Rev. Lett. 2014, 113, 175503.

[22] P. Wang, F. Casadei, S. Shan, J. C. Weaver, K. Bertoldi, Phys. Rev. Lett. 2014, 113, 014301.

[23] A. Rafsanjani, A. Akbarzadeh, D. Pasini, Adv. Mater. 2015, 27, 5931.

[24] J. T. Overvelde, T. Kloek, J. J. D’haen, K. Bertoldi, Proc. Natl. Acad. Sci. U.S.A. 2015, $112,10863$.

[25] D. Yang, B. Mosadegh, A. Ainla, B. Lee, F. Khashai, Z. Suo, K. Bertoldi, G. M.

Whitesides, Adv. Mater. 2015, 27, 6323.

[26] S. Timoshenko, J. M. Gere, Theory of Elasticity Stability, McGraw-Hill: New York, 1961.

[27] J. D. Madden, N. A. Vandesteeg, P. A. Anquetil, P. G. Madden, A. Takshi, R. Z. Pytel, S. R. Lafontaine, P. A. Wieringa, I. W. Hunter, IEEE J. Ocean. Eng. 2004, 29, 706.

[28] F. Daerden, D. Lefeber, Eur. J. Mech. Environ. Eng. 2002, 47, 11.

[29] G. K. Klute, J. M. Czerniecki, B. Hannaford, "McKibben Artificial Muscles: Pneumatic Actuators with Biomechanical Intelligence", presented at Proc. IEEE Int. Conf. Adv. Intell. Mechatron., 1999. 


\section{WILEY-VCH}

[30] C.-P. Chou, B. Hannaford, IEEE Trans. Robot. Autom. 1996, 12, 90.

[31] R. H. Baughman, Science 2005, 308, 63.

[32] G. Kovacs, L. Düring, S. Michel, G. Terrasi, Sens. Actuator A-Phys. 2009, 155, 299.

[33] Q. Pei, M. A. Rosenthal, R. Pelrine, S. Stanford, R. D. Kornbluh, "Multifunctional

Electroelastomer Roll Actuators and Their Application for Biomimetic Walking Robots", presented at Smart Structures and Materials, 2003.

[34] T. Mullin, S. Deschanel, K. Bertoldi, M. Boyce, Phys. Rev. Lett. 2007, 99, 084301.

[35] K. Bertoldi, M. Boyce, S. Deschanel, S. Prange, T. Mullin, J. Mech. Phys. Solids 2008, $56,2642$.

[36] M. Bowick, A. Cacciuto, G. Thorleifsson, A. Travesset, Phys. Rev. Lett. 2001, 87, 148103.

[37] R. Josephson, Annu. Rev. Physiol. 1993, 55, 527.

[38] M. Ikai, T. Fukunaga, Int. Z. Angew. Physiol. 1970, 28, 173. 
WILEY-VCH 
A Schematic representation of a VAMP
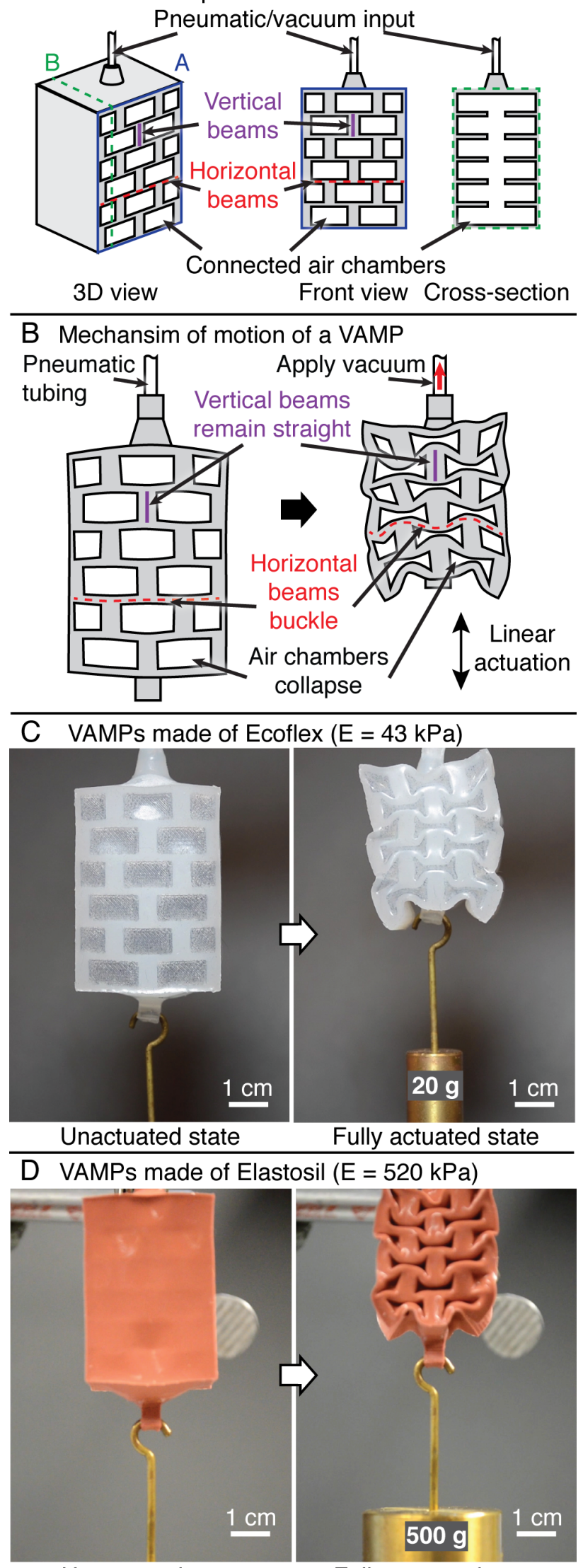

Unactuated state

Fully actuated state 


\section{WILEY-VCH}

Figure 1. Schematic description of VAMPs. (A) A VAMP consists of an elastomeric structure of vertical beams, thinner horizontal beams, and connected air chambers. The device is actuated using a single external port and a source of vacuum. (B) The vacuum allows ambient pressure to compress the structure, and thus to cause the thinner horizontal beams to buckle into serpentine shapes, which in turn causes the structure to compress anisotropically. (C) Images of a VAMP made of Ecoflex $(E=43 \mathrm{kPa})$ lifting a small weight $(20 \mathrm{~g})$ when actuated by applying a vacuum. The inside of the chamber membranes of this VAMP is colored with a black marker, such that the boundaries of the chambers are more visible in the actuated state. (D) A VAMP of the same geometry as in $(\mathrm{C})$, but made of Elastosil $(\mathrm{E}=520 \mathrm{kPa})$ lifts a much higher weight $(500 \mathrm{~g})$. As Elastosil is not transparent, the chambers of this VAMP are not visible in the unactuated state, but more visible in the actuated state as the membrane bends inwards. Scale bars are $1 \mathrm{~cm}$. See Movies S1 and S2 for the videos. Figure S2 and Movie S3 demonstrate VAMPs with even higher strength. 


\section{WILEY-VCH}
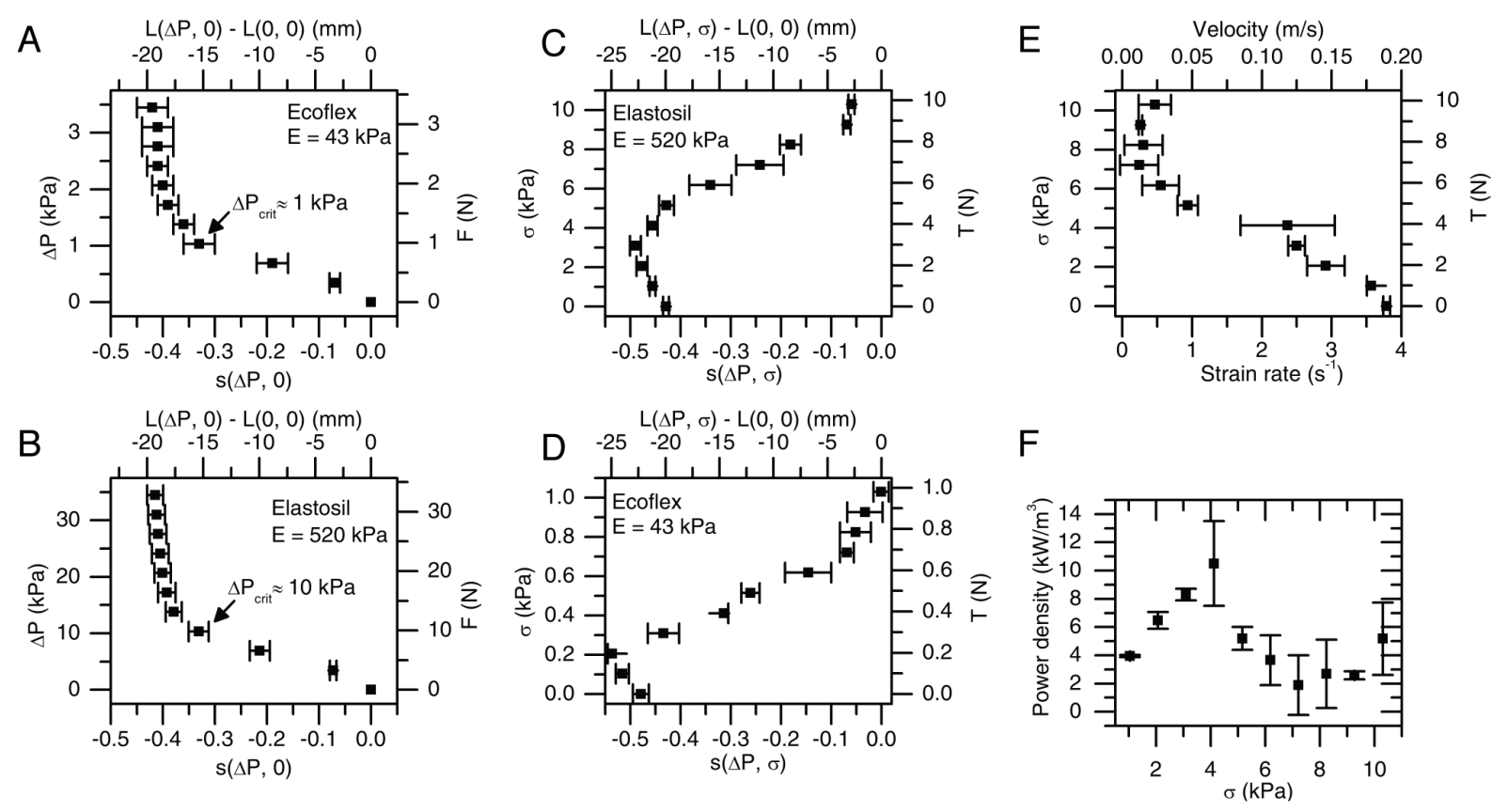

Figure 2. Static and dynamic characteristics of VAMPs. (A, B) Applied differential pressure $\Delta P$

vs. actuation strain $s(\Delta P, 0)$ curves of seven different VAMPs made of Ecoflex $(\mathrm{E}=43 \mathrm{kPa})$ and

Elastosil $(\mathrm{E}=520 \mathrm{kPa})$ at no load. The critical differential pressure $(\Delta P$ crit $)$ at which VAMPs

compress almost completely is proportional to the Young's modulus of the material. (C, D)

Loading stress $\sigma$ vs. actuation strain $s \Delta P, \sigma$ curves of seven different VAMPs made of Ecoflex (E

$=43 \mathrm{kPa})$ and Elastosil $(\mathrm{E}=520 \mathrm{kPa})$, when a differential pressure of $\Delta \mathrm{P}=90 \mathrm{kPa}$ is applied

(i.e. force-length relationship under active loading). A VAMP exerts greater force when

fabricated in a stiffer elastomer - an approximately ten-fold increase in the modulus of material

results in an approximately ten-fold increase in loading stress. (See the SI for discussions on the shape of the curve.) Reported values for static characteristics are mean \pm S.D. ( $n=7$ different samples). (In addition, $L \Delta P, \sigma-L 0, \sigma$ is the change in length of the VAMP, $F$ is the force due to difference of pressure, and $T$ is the total loading force.) (E) The relationship between force and 


\section{WILEY-VCH}

velocity for VAMPs fabricated in Elastosil. (F) The relationship between power density and stress for VAMPs fabricated in Elastosil. Reported values for dynamic characteristics are mean \pm S.D. $(\mathrm{n}=3$ measurements). 


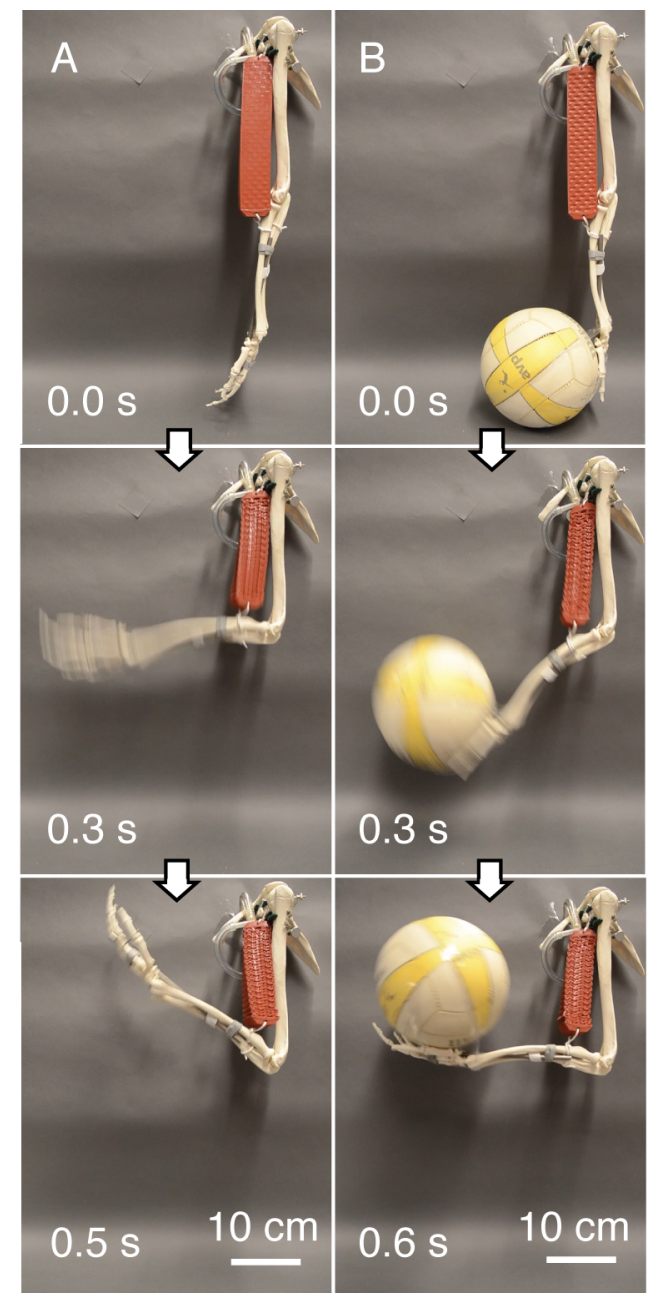

Figure 3. Demonstration of muscle-like actuation. (A) Use of a VAMP to actuate a polymer replica of the bones of a human arm, with mechanics similar to that employed by a human bicep muscle. Here metal wires serve as "tendons" to connect VAMP and replica bone, but high tensile strength polymer fibers could serve equally well. (B) The VAMP-actuated "arm" lifting a standard size and weight (274 g) volleyball. See Movies S4 and S5 for the videos. (See the SI, Figure S5, and Movies S6 and S7 for speed comparison with a fast-moving human arm.) 


\section{WILEY-VCH}

The mechanical features characteristics of biological muscles are difficult to reproduce completely in synthetic systems. We describes a new class of soft pneumatic structures (VAMPs, or vacuum-actuated muscle-inspired pneumatic structures) that combine actuation by negative pressure (vacuum), with cooperative buckling of beams fabricated in a slab of elastomer, to achieve motion and demonstrate many features that are similar to that of mammalian muscle.

\section{Keywords}

buckling, soft actuators, pneumatic actuation, linear actuator, muscle-like actuator.

D. Yang, M. S. Verma, J. So, B. Mosadegh, C. Keplinger, B. Lee, F. Khashai, E. Lossner, Z. Suo, and G. M. Whitesides*

\section{Buckling Pneumatic Linear Actuators Inspired by Muscle}

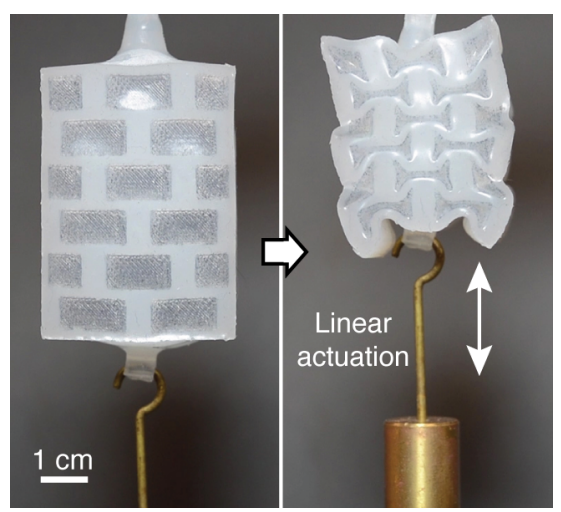

